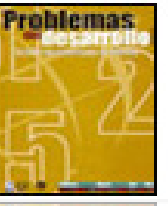

Problemas del Desarrollo. Revista Latinoamericana de Economía

ISSN: 0301-7036

revprode@servidor.unam.mx

Universidad Nacional Autónoma de México

México

Barrón Pérez, Antonieta

MICROFINANCIAMIENTO Y REDUCCIÓN DE LA POBREZA. FONDOS REGIONALES INDÍGENAS

Problemas del Desarrollo. Revista Latinoamericana de Economía, vol. 34, núm. 134, 2003, pp. 127-

148

Universidad Nacional Autónoma de México

Distrito Federal, México

Disponible en: http://www.redalyc.org/articulo.oa?id=11825944008

Cómo citar el artículo

- Número completo

- Más información del artículo

Página de la revista en redalyc.org

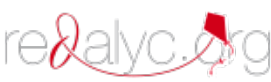

Sistema de Información Científica

Red de Revistas Científicas de América Latina, el Caribe, España y Portugal

Proyecto académico sin fines de lucro, desarrollado bajo la iniciativa de acceso abierto 


\title{
MiCROFINANCIAMIENTO Y REDUCCIÓN DE LA POBREZA. Fondos Regionales Indígenas
}

\section{Antonieta Barrón Pérez*}

Fecha de recepción: 22 de agosto de 2001. Fecha de autorización: 27 de noviembre de 2003.

\section{Resumen}

El propósito del presente trabajo es mostrar no sólo cómo se producen los apoyos del Programa de Fondos Regionales Indígenas a la población beneficiaria, sino presentar los desajustes e irregularidades, por las limitaciones en la normatividad y, en el peor de los casos, por su incumplimiento. A pesar de los desequilibrios que se observan en la región de estudio, donde el apoyo por tipo de proyecto es desigual, una hectárea de maíz se apoya con \$1273.00 y otra con $\$ 480.00$, además de que $30 \%$ de los fondos reciben $60 \%$ de los recursos. No obstante lo anterior, regulando la operación del Programa, su presencia entre los indígenas es indiscutible como un elemento de combate a la pobreza.

Palabras clave: indígenas, financiamiento, fondos regionales, Chiapas, política social.

\begin{abstract}
This work shows not only what kind of support the Regional Indigenous Funds gives its beneficiaries, but also the disadjustments and irregularities owing to limitations in the standards due in the worse of cases to lack of compliance. In spite of the imbalances observed in the region under study, where support for the different types of projects is unequal, some agriculturists, for example, receive $\$ 1273.00$ pesos per hectare of maize whereas others get $\$ 480.00$, apart from the fact that $30 \%$ of the Funds receive $60 \%$ of the resources. In spite of the above, once the Program's operation is regulated, its presence among the indigenous people will undoubtedly help struggle against poverty.
\end{abstract}

Key words: indigenous, financing, Regional Funds, Chiapas, social policy.

* Este trabajo es resultado de la evaluación hecha en 2000 a los Fondos Regionales Indígenas del estado de Chiapas en el que participaron José M anuel Hernández Trujillo y Ángel Enríquez Rangel.

** Profesora de la Facultad de Economía de la UNAM .Correos el ectrónicos: antonietabarron@ yahoo.com y mabarron@economia01.economia.unam.mx 


\section{Résumé}

L'objet de cet article est non seulement de montrer comment sont effectuées les aides du Programme de Fonds Régionaux Indiens à la population bénéficiaire, mais aussi de présenter leurs désajustements et leurs irrégularités, dûs aux limitations de la réglementation et, dans le pire des cas, à son manquement. Au-delà des déséquilibres observés dans la région étudiée, où l'aide est inégale selon le type de projet, une hectare de maïs bénéficie d'une aide de $\$ 1273.00$ et une autre de \$480.00; en outre, il a été observé que 30\% des Fonds reçoivent $60 \%$ des ressources. Cependant, nous croyons que si l'opération du Programme est régularisée, sa présence parmi les Indiens pourra s'affirmer comme un élément incontestable de combat contre la pauvreté.

Mots-cléfs: indiens, financement, Fonds régionaux, Chiapas, politique sociale.

\section{Resumo}

O propósito deste trabalho é o de mostrar não só como se produzem os apoios do programa de Fundos Regionais Indígenas à população beneficiaria, mas também apresentar os desajustes e irregularidades, pelas limitações na normatividade e, no pior dos casos, pelo seu não cumprimento. A pesar dos desequilíbrios observados na região em estudo, onde o apoio por tipo de projeto é desigual, uma hectare de milho recebe $\$ 1273,00$ e outra $\$ 480,00$. Além do mais, 30\% dos Fundos recebem $60 \%$ dos recursos. Não obstante o anterior, regulando a operação do programa, a sua presença entre os indígenas é indiscutível como um elemento de combate à pobreza.

Palavras chave: indígenas, financiamento, Fundos regionais, Chiapas, política social. 


\section{Introducción}

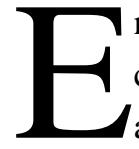

n México, según el Censo de Población de 2000, había 7278002 de indígenas, cifra que creció a una tasa menor respecto a la población total, 1.27\% promedio anual entre 1990 y 2000. La población de cero a cuatro años creció a una tasa de $0.98 \%$, es posible suponer que ello se deba a la precariedad de sus condiciones de vida. En términos absolutos, hay aproximadamente 866 mil indígenas más en el país. La Encuesta Nacional de Empleo en Zonas Indígenas (ENEZI) ${ }^{1}$ estimó, para 1997, que había aproximadamente 10 millones de indígenas ${ }^{2}$ de los cuales 6.5 millones eran de doce años y más y, de éstos, aproximadamente 4 millones son población ocupada. Según la ENEZI, de la población ocupada total, $87 \%$ realiza actividades agropecuarias. ${ }^{3}$ El predominio de esta actividad es una constante en todos aquellos lugares donde se asienta la población indígena, incluyendo a ésta entre los grupos de población en condiciones de pobreza extrema, sujetos, en el mejor de los casos, a los beneficios de la política social.

La población indígena no sólo vive en extrema pobreza, sino que sus condiciones de trabajo están más deterioradas que el promedio de la población ocupada del país. En 1997, según la ENEZI, 47.7\% de esta población no recibió ingresos o los obtuvo de forma no monetaria y $32.5 \%$ obtuvo menos de un salario mínimo. En promedio para el país, en ese mismo año, a diferencia de la población total, $15.1 \%$ no obtiene ingresos y $21 \%$, menos de un salario mínimo. Como se puede ver, los indígenas son los más pobres entre los pobres.

\section{El lugar de Chiapas en el contexto nacional}

Ocho entidades absorben $65.2 \%$ de la población indígena de cinco años y más: Oaxaca, Chiapas, Veracruz, Puebla, Yucatán, Guerrero, México e Hidalgo; Chiapas ocupa el segundo lugar, con $11.1 \%$ de la población indígena, cuyas características de la fuerza de trabajo son las siguientes.

Según la ENEZI, ${ }^{4}$ en Chiapas existen 379714 indígenas de 12 años y más, de los cuales 66.5\% son económicamente activos. En 1997 la población ocupada ascendía a 250999 personas, de las cuales $83.6 \%$ se encontraba en el sector agropecuario, como ocupación

Única encuesta levantada para población indígena.

2 Las diferencias con el censo de población de INEGI se debe a que en la ENEZI se trata de estimaciones.

3 INEGI-INI, Encuesta Nacional de Zonas Indígenas, 1997.

4 Se toma como base la ENEZI porque presenta información más agregada por entidad, a diferencia del Censo de Población aunque éste sea más actual. 
principal $83.5 \%$ son trabajadores agropecuarios y por posición en el trabajo $12.2 \%$ son asalariados y $43.6 \%$ no lo son, la mayoría labora por cuenta propia. Es una población que genera su propio trabajo ante la precariedad del mercado en la entidad.

Como la única opción de financiamiento para la población indígena fueron creados los Fondos Regionales Indígenas. ${ }^{5}$ No obstante, existen otros programas desde principios de la década de los noventa a los cuales no tienen acceso.

Según estadísticas oficiales, existen 42 millones de pobres en el país, de los cuales entre 8 y 10 millones tienen potencialidades para desarrollarse como microempresarios. Adicionalmente, de acuerdo con los censos económicos, hay 3 millones de empresas, de las cuales 2.8 millones son micro y pequeñas empresas y de éstas sólo 100 mil tienen acceso a algún crédito por parte del sistema bancario, ${ }^{6}$ lo que significa que apenas $3.6 \%$ ha podido tener el beneficio de algún tipo de financiamiento.

$\mathrm{Si}$ agregamos que la mayoría de los beneficiados proviene de zonas urbanas, entonces resulta importante la presencia de los Fondos Regionales Indígenas como opción de financiamiento en zonas rurales y además, indígenas.

Bajo estas consideraciones, el propósito del presente artículo es analizar el funcionamiento de los citados fondos en Chiapas y su impacto entre la población beneficiaria, a partir de una evaluación externa hecha en el año 2000. Pese a la fecha, el trabajo mantiene vigencia al cambiar el Instituto Nacional Indigenista (INI) por la Comisión Nacional para el Desarrollo de los Pueblos Indígenas, momento en que puede corregirse el desempeño de los programas de apoyo dirigido a esta población.

Se hace énfasis en dos aspectos que han sucedido con la mecánica de promover proyectos rentables: que impulsen la diversificación productiva, eleven la productividad y generen empleo e ingresos regulares.

\section{Origen y propósitos de los Fondos Regionales Indígenas}

Con la creación del INI en 1948, se desarrolla un organismo que tiene como propósito determinar las políticas de atención a los pueblos indígenas, pero es hasta 1971 que se encarga de la instrumentación de las acciones del Programa Integral de Desarrollo Rural (PIDER), con el cual se comienza a implantar una estrategia de desarrollo comunitario, basado en tres líneas fundamentales de intervención: un programa de salud, uno de abasto comunitario y otro de construcción y mejoramiento de vivienda.

Posteriormente, en junio de 1986, con el fin de que se desarrollaran proyectos productivos autónomos y autogestivos en las comunidades indígenas se aprobó el reglamento

5 LOS FRI, surgieron en el Instituto Nacional Indigenista, hoy Comisión Nacional para el Desarrollo de Pueblos Indígenas, donde se agregaron al programa de Pueblos y Comunidades Indígenas, pero continúa con las mismas funciones.

6 Tomado de www.cgap.org/docs/CaseStudy_centralamerica 
para la constitución del Fondo Comunitario de Proyectos Productivos de las Comunidades Indígenas. Los recursos de este fondo fueron manejados por los núcleos y comunidades beneficiadas, organizados en los Comités Comunitarios de Planeación (COCOPLA).

El 19 de marzo de 1990, el presidente Carlos Salinas de Gortari anunció la creación de 100 Fondos Regionales de Solidaridad (FRS, hoy FRI), ${ }^{7}$ con un monto inicial de N\$500 000 cada uno. Cuando el ex mandatario dio inicio al programa dejó en claro que "el Instituto (Nacional) Indigenista no intervendrá en las decisiones que ustedes tendrán que tomar para la asignación de los Fondos de Solidaridad", ${ }^{8}$ la idea, aunque no escrita, era darle continuidad a los COCOPLA, pero no se logró.

A partir de ese año, el gobierno federal aportaría dotaciones anuales de capital a las organizaciones indígenas a través de los FRI. Los recursos serían asignados por el entonces Programa Nacional de Solidaridad y estarían destinados al financiamiento de proyectos productivos autosostenibles, rentables y recuperables. ${ }^{9}$

Así, los Fondos Regionales Indígenas se constituyen en organizaciones regionales de indígenas con estructura y patrimonio propios, con los fines mencionados anteriormente y cuyos objetivos generales son:

- Promover la participación activa de las comunidades indígenas en la programación, presupuestación, ejecución, seguimiento y evaluación de los proyectos, mediante el fortalecimiento de los procesos organizativos.

- Crear una agrupación de organizaciones y comunidades en cada una de las regiones indígenas que actúe como interlocutor de todas las acciones que realiza el Instituto y otras dependencias del sector público, tanto federales como estatales.

y los específicos:

- Fortalecer la autonomía de las organizaciones y comunidades indígenas con el fin de que manejen, de manera directa e independiente, sus recursos.

- Lograr que las organizaciones y comunidades citadas participen activamente en la planeación de todos los proyectos orientados a su desarrollo.

- Fortalecer procesos organizativos en las comunidades con el propósito de evitar la concentración de recursos en organizaciones que, por lo general, tienen acceso a otras fuentes de financiamiento.

- Establecer proyectos productivos con criterios de rentabilidad, sobre la base de una verdadera corresponsabilidad con las comunidades indígenas.

7 En la administración de Ernesto Zedillo le cambiaron el nombre a Fondos Regionales Indígenas (FRI, en adelante se usarán estas siglas). Su función no cambió actualmente con la desaparición del INI y la creación de la Comisión Nacional para el Desarrollo de los Pueblos Indígenas.

8 INI-SEDESOL, Instituto Nacional Indigenista 1989-1994, M éxico, 1994.

9 Op. cit., p. 99. 
- Impulsar la diversificación productiva y elevar la productividad de los pueblos indígenas mediante la entrega de recursos y la capacitación de sus integrantes.

- Lograr el reconocimiento formal de las figuras asociativas que las comunidades determinen para que tengan acceso a las distintas fuentes de financiamiento existentes.

- Propiciar que los beneficios derivados de las acciones productivas tiendan a capitalizar a las organizaciones y comunidades indígenas.

- Generar más empleos en las comunidades que redunden en mejores condiciones de vida de su población. ${ }^{10}$

De los objetivos generales y particulares en una década, unos no se cumplieron y otros de manera parcial, pero el impacto inmediato de ofrecer financiamiento a los indígenas en Chiapas se cumplió, sin embargo, no llegó en la forma que marca la normatividad y se distribuyó con la limitación que marca el capital disponible y el control del mismo.

\section{Estructura organizativa y normatividad}

\section{Estructura organizativa}

LOS FRI se conformaron a partir de la convocatoria que llevaron a cabo los Consejos Coordinadores Indigenistas (CCI) a todas las organizaciones de la región para conformar la Asamblea General de cada Fondo.

De acuerdo con las reglas de operación propuestas por el INI, la Asamblea General de representantes constituye el máximo órgano de decisión del fondo y se integra por los delegados electos en el seno de sus organizaciones.

Cada FRI cuenta con un Consejo Directivo nombrado por la Asamblea General de representantes, el cual dura dos años en su puesto y no existe la posibilidad de reelección en el periodo inmediato. El cargo es honorífico y no tiene remuneración económica, aunque se proporcionan apoyos financieros para gastos de transportación, hospedaje y alimentación, cuando su trabajo así lo requiere. Todos los consejos directivos de Fondos tienen al menos tres comisiones: financiera, técnica y de evaluación. Además cuenta con un secretariado técnico, que está compuesto por el personal directivo, técnico y administrativo de los CCI y por los módulos de apoyo. Su función sólo consiste en orientar, acompañar, apoyar y asesorar a las organizaciones, por lo que respeta, invariablemente, sus acuerdos y decisiones. Este secretariado es corresponsable con los FRI de la ejecución adecuada del programa y de realizar el traspaso de los recursos financieros hacia las organizaciones indígenas. En las reuniones de las diversas instancias del fondo tiene derecho a voz, pero no a voto.

10 SEDESOL-INI-SOLIDARIDAD, Fondos Regionales de Solidaridad (M anual de Operación), M éxico, INI, 1993, pp. 10-11. 
Los módulos de apoyo están integrados por técnicos cuya función es dar asesoría, apoyo y capacitación específica a los FRI y a sus organizaciones en aspectos administrativos, contables y técnicos que el secretariado técnico no pueda proporcionar.

\section{Normatividad para el manejo de los recursos}

En la normatividad de los FRI se consideraba que los recursos deberían de estar orientados a financiar proyectos productivos, rentables, autosostenibles y además, tener un amplio número de beneficiarios. Asimismo, deberían responder a una demanda de la organización promotora y tender a dinamizar procesos productivos más amplios. Bajo estas consideraciones, el propósito del programa es apoyar a una población lo más amplia posible. Para ello se estableció una regla que señalaba que ningún proyecto debería recibir más de $20 \%$ del capital total del fondo ni $10 \%$ si la organización tuviese otros proyectos en ejecución, lo anterior, con el propósito de evitar la concentración de recursos en manos de las organizaciones más fuertes.

Para recibir financiamiento, las organizaciones deberán presentar un expediente técnico que muestre la justificación del mismo, la mecánica operativa, el desglose presupuestal, un calendario de ministraciones, el número de beneficiarios, su impacto social, recuperaciones y copia del acta de aprobación del proyecto por parte de la asamblea general de la organización o comunidad solicitante.

La normatividad de transferencia de recursos a los fondos ha sufrido algunos cambios que se derivan de la fuente de recursos, entre los más importantes se encuentran los siguientes:

- En mayo de 1990, el Consejo Directivo del INI aprobó una normatividad provisional para la operación de los FRI, concebida como un instrumento flexible y adaptable a las condiciones particulares de cada una de las regiones indígenas, pero también riguroso en el manejo honesto y transparente de los recursos económicos. ${ }^{11}$

- Durante 1993 y primer semestre de 1994, el programa operó con la normatividad establecida en el ramo xxvi, Solidaridad y Desarrollo Regional del Presupuesto de Egresos de la Federación. Con ello, la autorización de los proyectos propuestos por las organizaciones y aprobados por las asambleas generales de representantes de los FRI, quedó en manos de la SEDESOL. Esto implicó que la aprobación de los proyectos estuviera sujeta a un complicado entramado administrativo, lo que ocasionó que en 1993 los recursos llegaran a las comunidades a destiempo, esto es, fuera de los ciclos agríco-

11 SEDESOL-INI-SOLIDARIDAD, Instituto Nacional, p. 102. 
las. Por este motivo, $80 \%$ de los proyectos aprobados por la SEDESOL tuvieron que ser reformulados por las organizaciones indígenas. ${ }^{12}$

- A partir de junio de 1994, las organizaciones deben presentar sus proyectos y anexos técnicos para que la Dirección General de Planeación de la SEDESOL emita los oficios de autorización a nombre del INI, y a favor de los FRI correspondientes. Una vez que los proyectos son autorizados, se envían los recursos financieros al INI y se distribuyen a través del Centro Coordinador Indigenista (CCI), quienes cuentan con cinco días hábiles para transferirlos a los consejos directivos de los FRI. ${ }^{13}$

- El documento legal que ampara la comprobación es el Convenio de Transferencia suscrito por el INI y el Consejo Directivo de los FRI correspondientes, avalado por la asamblea general de representantes.

Pese a las modificaciones en la normatividad para asignar apoyos, en la práctica, los consejos directivos y las propias asambleas han transgredido la normatividad. En todos los fondos de Chiapas no es poco frecuente que el proyecto se presente en forma muy simple y sin anexo técnico. Presentarlo de esta forma obedece, en la mayoría de los casos, a las características de los proyectos apoyados, como compra de fertilizante, de una acémila, por mencionar algunos. Los menos, sí requieren la presentación del anexo mencionado. Lo que se mantiene es someter a concurso la compra del bien determinado, ya sea una vaca o una despulpadora de café.

\section{Funcionamiento de los Fondos Regionales Indígenas}

Distribución del presupuesto por fondo

En 1997, en la entidad había dieciséis Fondos Regionales Indígenas. Para 1999 existían veinticuatro - $\mathrm{COESCO}^{14}$ desapareció en 1997. La norma es que el Fondo se ubique en un radio en el cual las comunidades beneficiadas se localicen a una distancia suficiente como para que las organizaciones puedan trasladarse con cierta facilidad y lo hacen, regularmente, en un sitio que opera como centro político regional.

No hay más lógica para la asignación del presupuesto que las propuestas por los socios de cada Fondo para la capitalización al año siguiente. Aunque formalmente se entrega una propuesta de cómo se usarán los fondos, en ningún momento se les plantea qué hicieron con las recuperaciones.

Como puede observarse en el Cuadro 1, después de 1995, el presupuesto federal ejercido por los fondos aumentó en forma desorbitada. Sin embargo, la distribución desequi-

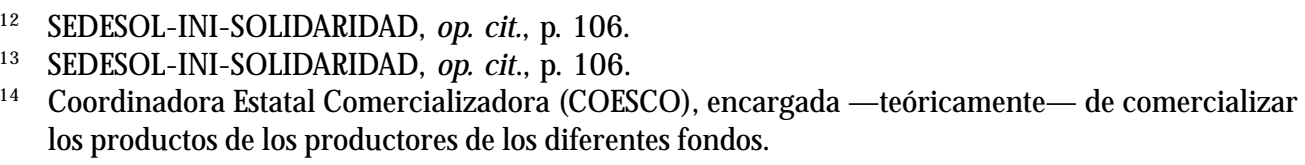

14 Coordinadora Estatal Comercial izadora (COESCO), encargada - teóricamente- de comercializar los productos de los productores de los diferentes fondos. 
Cuadro 1

Presupuesto federal ejercido por Fondo Regional Indígena

(en pesos corrientes)

\begin{tabular}{|c|c|c|c|c|c|}
\hline Fondo & 1995 & 1996 & 1997 & 1998 & 1999 \\
\hline COESCO & 344721 & 5773026 & 4937080 & 0 & 0 \\
\hline Teopisca & 572000 & 550768 & 825700 & 1336700 & 1621940 \\
\hline Bochil & 0 & 5600556 & 7414320 & 6353444 & 5999998 \\
\hline San Andrés Duraznal & 0 & 0 & 0 & 0 & 1200000 \\
\hline Las Margaritas & 1220024 & 4373026 & 5642502 & 5495390 & 6200000 \\
\hline Maravilla Tenejapa & 0 & 0 & 0 & 0 & 1000000 \\
\hline San Quintín & 318600 & 768090 & 1569471 & 982100 & 1104600 \\
\hline Taniperlas & 501950 & 1177734 & 907795 & 1083015 & 1199971 \\
\hline Sitalá & 1522775 & 562359 & 949009 & 861768 & 1493086 \\
\hline Ocosingo & 2431057 & 1480173 & 785970 & 1082679 & 1198225 \\
\hline Ixtacomitán & 700437 & 5305621 & 2820254 & 4714882 & 5089045 \\
\hline Region Chóol. Tila & 190920 & 896834 & 667005 & 4031867 & 2500000 \\
\hline Sabanilla & 0 & 0 & 0 & 0 & 1209310 \\
\hline San Cristóbal de las Casas & 1539700 & 1961468 & 1487073 & 2028427 & 2975909 \\
\hline Aldama & 0 & 0 & 0 & 0 & 744716 \\
\hline Mujeres indígenas & 0 & 0 & 0 & 0 & 398753 \\
\hline Santiago El Pinar & 0 & 0 & 0 & 0 & 1070869 \\
\hline Copainalá & 0 & 2298924 & 2965928 & 3112644 & 3657643 \\
\hline Venustiano Carranza & 0 & 7322518 & 8711097 & 8264893 & 6000000 \\
\hline Ocozocoautla & 0 & 4488166 & 3717375 & 5149998 & 5098756 \\
\hline Mazapa de Madero de la Sierra & 0 & 4550708 & 5153278 & 5665066 & 4769392 \\
\hline Santo Domingo & 0 & 2536784 & 1695500 & 3159346 & 1875500 \\
\hline Palenque & 0 & 5370935 & 6058551 & 6595197 & 5999886 \\
\hline Marqués de Comillas & 0 & 2212983 & 1730277 & 2251907 & 1800000 \\
\hline Amatán & 0 & 1390122 & 1069027 & 2639249 & 1978517 \\
\hline Total & 9342184 & 58620795 & 59107212 & 64808572 & 66186116 \\
\hline
\end{tabular}

Fuente: INI, Departamento de Estadísticas.

librada entre éstos obedeció a dos razones: la capacidad para negociar de sus dirigentes y los conflictos políticos de la zona; en mucho menor medida se consideró el número de organizaciones asociadas al fondo.

En 1995, por ejemplo, tres Fondos — Las Margaritas, Sitalá y Ocosingo— absorbieron 55.4\% del presupuesto federal ejercido. De 1996 a 1999, sólo Las Margaritas mantuvo en forma creciente su presupuesto y en 1999 ya se observa una distribución más equitativa.

De acuerdo con la normativa, después de los primeros años de iniciado el Programa Fondos, en el cual a cada uno se le asignaban $\$ 500$ 000, en Chiapas los montos variaron, ${ }^{15}$ sobre todo, a partir de 1996. No se explica por qué el presupuesto per cápita sea tan desigual entre fondo o por tipo de proyecto.

15 No se tienen elementos para suponer que en fondos de otras entidades las cosas fueron diferentes, además, no es nuestro propósito. 
Cuadro 2

Presupuesto total ejercido y para siembra de maíz por Fondo en Chiapas, 1999

\begin{tabular}{|c|c|c|}
\hline & \multicolumn{2}{|c|}{ Asignaciones Promedio } \\
\hline & Total & Siembra Maíz \\
\hline FRI. & $\$$ per cápita & $\$$ por ha \\
\hline Delegación estatal & 1298.59 & - \\
\hline Teopisca & 1298.59 & 1273.42 \\
\hline Bochil & 1535.84 & 628.95 \\
\hline San Andrés Duraznal & 2696.63 & - \\
\hline Las Margaritas & 877.07 & 598.13 \\
\hline Maravilla Tenejapa & 1788.91 & \\
\hline San Quintín & 2789.39 & - \\
\hline Taniperlas & 4363.53 & 581.95 \\
\hline Sitalá & 965.77 & 661.67 \\
\hline Ocosingo & 1350.87 & 893.37 \\
\hline Ixtacomitán & 8065.05 & - \\
\hline Region Chóol. Tila & 2732.24 & 498.23 \\
\hline Sabanilla & 3620.69 & \\
\hline San C. de las Casas & 2975.91 & 891.99 \\
\hline Aldama & 1251.62 & - \\
\hline Mujeres indígenas & 674.71 & - \\
\hline Santiago El Pinar & 2759.97 & - \\
\hline Copainalá & 10726.23 & - \\
\hline Venustiano Carranza & 2155.17 & 1213.71 \\
\hline Ocozocoautla & 4369.11 & 931.05 \\
\hline Mazapa de Madero de la Sierra & 2244.42 & 1467.48 \\
\hline Santo Domingo & 9768.23 & - \\
\hline Palenque & 11256.82 & - \\
\hline Marqués de Comillas & 7377.05 & - \\
\hline Amatán & 6182.87 & 1186.26 \\
\hline Total & 2305.81 & \\
\hline
\end{tabular}

Fuente: Elaborado con base en los datos de INI.

Pudiera pensarse que los ingresos asignados por Fondo sean desiguales, por tratarse de proyectos diferentes, unos más costosos que otros y diferente número de beneficiarios; sin embargo, cuando analizamos las asignaciones por hectárea para la producción de maíz, encontramos marcadas distinciones, lo que muestra la falta de criterios entre los diferentes FRI.

No es posible que entre fondos haya disimilitudes tan grandes para explotar una hectárea de maíz. Por lo general, los apoyos a la producción de cultivo significan compra de fertilizante, algunas veces incluyen bombas aspersoras de éste, no obstante, eso no explica la disparidad en la asignación de recursos por hectárea, aun cuando se justificaran las diferencias por persona, debido a la posible variedad en el tamaño del predio.

Pese a que existe una normatividad, según se observa en los reportes, no parece haber un criterio para la asignación de recursos por tipo de proyecto. Su falta de control provocó 
Cuadro 3

Presupuesto ejercido por fondo y productores beneficiados. 1999

\begin{tabular}{|c|c|c|c|}
\hline Proyecto & $\begin{array}{l}\text { Presupuesto } \\
\text { ejercido* }^{*}\end{array}$ & $\begin{array}{l}\text { Número de } \\
\text { productores }\end{array}$ & $\begin{array}{l}\text { Asignación } \\
\text { media }\end{array}$ \\
\hline Delegación estatal & 1621940.00 & 1249.00 & 1298.59 \\
\hline Teopisca & 1621940.00 & 1249.00 & 1298.59 \\
\hline Bochil & 5999998.00 & 4243.00 & 1414.09 \\
\hline San Andrés Duraznal & 1200000.00 & 445.00 & 2696.63 \\
\hline Las Margaritas & 6200000.00 & 6510.00 & 952.38 \\
\hline Maravilla Tenejapa & 1000000.00 & 559.00 & 1788.91 \\
\hline San Quintín & 1104600.00 & 396.00 & 2789.39 \\
\hline Taniperlas & 1199971.00 & 275.00 & 4363.53 \\
\hline Sitalá & 1493086.00 & 1546.00 & 965.77 \\
\hline Ocosingo & 1198225.00 & 887.00 & 1350.87 \\
\hline Ixtacomitán & 5089045.00 & 631.00 & 8065.05 \\
\hline Región Cho’ol & 2500000.00 & 915.00 & 2732.24 \\
\hline Sabanilla & 1209310.00 & 334.00 & 3620.69 \\
\hline San Cristóbal de las Casas. Los Altos & 2975909.00 & 1000.00 & 2975.91 \\
\hline Aldama & 744716.00 & 595.00 & 1251.62 \\
\hline Mujeres indígenas & 398753.00 & 591.00 & 674.71 \\
\hline Santiago El Pinar & 1070869.00 & 388.00 & 2759.97 \\
\hline Copainalá & 3657643.00 & 341.00 & 10726.23 \\
\hline Venustiano Carranza & 6000000.00 & 2784.00 & 2155.17 \\
\hline Ocozocoautla & 5098756.00 & 1167.00 & 4369.11 \\
\hline Mazapa de Madero. La Sierra & 4769392.00 & 2125.00 & 2244.42 \\
\hline Santo Domingo & 1875500.00 & 192.00 & 9768.23 \\
\hline Palenque & 5999886.00 & 533.00 & 11256.82 \\
\hline Marqués de Comillas & 1800000.00 & 244.00 & 7377.05 \\
\hline Amatán & 1978517.00 & 320.00 & 6182.87 \\
\hline Total & 65185116.00 & 28270.00 & 2305.81 \\
\hline
\end{tabular}

Fuente: INI.

* pesos corrientes.

que en algunas organizaciones, un miembro de la mesa directiva, del secretariado del fondo, del módulo de apoyo o el contador concentrara el poder y mantuviera el manejo de los recursos.

En cuanto al control de éstos, no se logró que las organizaciones participantes se apropiaran del programa de fondos y su distribución se hizo más inequitativa con los años, como puede verse en el Cuadro 3.

Si analizamos qué tipo de distribución hicieron los fondos en los cuales las asignaciones se disparan, encontramos una limitante. Cuando se apoyó un proyecto para una comunidad o varias, la forma de registro impide saber la magnitud del proyecto, como por ejemplo, la rehabilitación de potreros.

Otro fenómeno que lleva a la duda sobre el manejo de los recursos es que en un mismo año, el pago por una vaca de engorda varía según la región, ya sea que se trate, en efecto, de una de este tipo o de doble propósito. En este caso, no sólo es la discrecionalidad en el 
Cuadro 4

Destino de los recursos asignados en los fondos donde se disparan las asignaciones, 1999

\begin{tabular}{lrrrr}
\hline & $\begin{array}{r}\text { Presupuesto } \\
\text { federal ejercido }\end{array}$ & $\begin{array}{c}\text { Unidad de } \\
\text { medida }\end{array}$ & $\begin{array}{r}\text { Asignación } \\
\text { media por } \\
\text { proyecto }\end{array}$ \\
\hline TANIPERLAS & 1199971.00 & & & \\
Adquisición y distribución de fertilizante p/maíz & 155380.00 & Hectárea & 227 & 684.49 \\
Unidad bovina de engorda & 974600.00 & Cabeza & 278 & 3505.76 \\
Rehabilitación de potreros & 69991.00 & Proyecto & 1 & 69991.00 \\
IXTACOMITÁN & 5089045.00 & & \\
Unidades bovinas de doble propósito & 3158530.00 & Cabeza & 715 & 4417.52 \\
Unidades bovinas de engorda & 1950515.00 & Cabeza & 654 & 2982.44 \\
COPAINALÁ & 3657643.00 & & & \\
Unidades bovinas de doble propósito & 2276536.00 & Cabeza & 394 & 5778.01 \\
Unidades bovinas de engorda & 1323464.00 & Cabeza & 456 & 2902.33 \\
Unidades bovinas de engorda & 57643.00 & Cabeza & 20 & 2882.15 \\
PALENQUE & 5999886.00 & & & \\
Unidades bovinas de engorda & 4869465.00 & Cabeza & 1593 & 3056.79 \\
Acopio y comercialización de café & 600000.00 & Proyecto & 1 & 600000.00 \\
Acopio y comercialización de pescado & 530421.00 & Proyecto & 1 & 530421.00 \\
AMATÁN & 1978517.00 & & & \\
Adquisición de insumos para cultivo básico & 148282.00 & Hectárea & 125 & 186.26 \\
Adquisición de máquinas despulpadoras & 28098.00 & Proyecto & 1 & 28098.00 \\
Unidades bovinas de engorda & 165310.00 & Cabeza & 54 & 3061.30 \\
Unidades bovinas de engorda & 811285.00 & Cabeza & 240 & 3380.35 \\
Acopio y comercialización de café & 168285.00 & Proyecto & 1 & 168285.00 \\
Unidades bovinas de doble propósito & 657165.00 & Cabeza & 124 & 5299.72 \\
\hline
\end{tabular}

Fuente: Departamento de Estadísticas del INI.

manejo del recurso, sino que las licitaciones no operan como es debido ${ }^{16}$ ni se busca la compra en volumen para negociar el precio a la baja.

Lo anterior da como resultado que el Consejo Directivo y los beneficiarios no estén conscientes de que el dinero que les llegó es de ellos y deben cuidarlo, para capitalizarse.

Pero aun si no hubiera irregularidades en las asignaciones, la mayoría de los apoyos, por el monto asignado, no puede ser opción de proyecto productivo.

Como podemos observar en el Cuadro 5, entre 1995 y 1999, en el caso de la agricultura, los montos asignados por tipo de proyecto no permiten suponer que se trata de proyectos rentables - aun cuando sean productivos- y en el caso de los pecuarios, la imposibilidad de considerarlos de este tipo estriba en que se trata de proyectos individuales y no de grupo. El sentimiento de propiedad de los indígenas poseedores de una vaca les impide asociarse para la reproducción y venta del ganado.

Adicionalmente, la política de que hay qué beneficiar al mayor número de socios impide volver rentable un proyecto, a cada socio se le da apoyo por una sola vez y no se le vuelve a otorgar hasta que no lo haya recibido el resto de los socios. En la práctica, cada

16 No llegan los oferentes con sobre cerrado y se dan casos en que sólo llega uno.

\section{Desaarrrollo}


Cuadro 5

Asignaciones medias por beneficiario por tipo de proyecto apoyado

\begin{tabular}{|c|c|c|c|}
\hline \multicolumn{3}{|c|}{$\begin{array}{c}1995 \\
\text { \$ por beneficiario }\end{array}$} & \multirow[t]{2}{*}{$\begin{array}{c}1999 \\
\text { \$ por beneficiario }\end{array}$} \\
\hline 01. Agrícola & & 01. Agrícola & \\
\hline Apoyo a la producción de maíz & 878.85 & Adquisición de bombas aspersoras & 211.25 \\
\hline Apoyo al cultivo de café & 500.76 & Cultivo de trigo & 358.62 \\
\hline Adquisición de bombas aspersoras & 322.95 & Cultivo de papaya & 6296.36 \\
\hline Adquisición de despulpadoras & 750.87 & Cultivo de jenjibre & 708.77 \\
\hline Apoyo al cultivo de frijol & 718.85 & Cultivo de piña & 1063.63 \\
\hline Producción de papa & 8971.28 & Producción de papa & 4014.54 \\
\hline Cultivo de achiote & 526.72 & Fertilizantes para maíz & 1363.08 \\
\hline Producción de chayotes & 1900.35 & Producción de flor & 4834.22 \\
\hline Mecanización agrícola & 674.59 & Fertilizante para café & 1323.71 \\
\hline Cultivo de melón & 9035.67 & 02. Pecuario & \\
\hline Producción de chile & 2000.00 & Criadero y dotación de acémilas & 5996.15 \\
\hline 02. Pecuario & & Infraestructura pecuaria & 2200.00 \\
\hline Ganado bovino de engorda & 3085.05 & Instalación de apiarios & 4732.81 \\
\hline Dotación de acémilas & 1857.78 & Rehabilitación de potreros & 2499.68 \\
\hline Alimento para ganado porcino & 2142.86 & Unidad avícola de engorda & 1988.68 \\
\hline Rehabilitación de potreros & 2288.89 & Unidad bovina de doble propósito & 5974.60 \\
\hline Instalación de granjas de pavo & 1500.00 & Unidad bovina de engorda & 7648.51 \\
\hline Bovino de doble propósito & 4203.01 & Unidad bovina de reproducción & 7121.07 \\
\hline Yuntas bovinas & 8240.00 & Unidad ovina de reproducción & 4005.76 \\
\hline Asistencia técnica apícola & 2593.65 & Unidad porcina de engorda & 2806.10 \\
\hline Otros & 624.79 & Otros & 1247.94 \\
\hline
\end{tabular}

Fuente: Departamento de Estadística del INI.

Consejo Directivo abusa de su poder y se autopresta más ocasiones, no importando que no haya pagado la deuda anterior.

\section{Resultados de los apoyos}

Los mecanismos para aprobar proyectos y para recuperar los recursos se siguen formalmente como lo marca la norma, sin embargo, hay dos conjuntos de fenómenos que impiden las recuperaciones. En el primero se encuentran las formas de organización de los grupos para los apoyos, se junta uno de quince o cincuenta personas, o firman los jefes de familia de un pueblo, por lo que no existe un responsable. Debido a la asignación del proyecto, como la compra de un camión, que es el fenómeno más recurrente, propicia que no haya pago y que la concentración del proyecto recaiga en una sola persona.

En el otro conjunto de problemas se encuentra la cultura del no pago. Los indígenas no han entendido que el dinero es de ellos y que deben recuperarlo para poder capitalizarse, muchos no pagan porque están convencidos de que el dinero se los deben, otros pagan e inmediatamente vuelven a pedir prestada la misma cantidad, pasando por alto los acuerdos de la Asamblea. 
Cuadro 6

Proyectos vencidos

\begin{tabular}{lrrr}
\hline \multicolumn{1}{c}{ Rama } & Total & Vencido & $\%$ \\
\hline Básicos & 100 & 56 & 56.0 \\
Frutícolas & 8 & 3 & 37.5 \\
Hortalizas & 11 & 9 & 81.8 \\
Flores & 2 & 1 & 50.0 \\
Otros agrícolas & 11 & 7 & 63.6 \\
Bovino & 29 & 8 & 27.6 \\
Porcino & 1 & 1 & 100.0 \\
Aví́cola & 1 & 1 & 100.0 \\
Apícola & 2 & 2 & 100.0 \\
Otro ganado & 10 & 7 & 70.0 \\
Pesca & 2 & 2 & 100.0 \\
Deshidratados & 9 & 8 & 88.8 \\
Tortillería & 3 & 2 & 66.6 \\
Construcción de caminos & 1 & 0 & 0.0 \\
Textiles & 2 & 0 & 0.0 \\
Artesanías & 2 & 1 & 50.0 \\
Transporte & 3 & 2 & 66.6 \\
Tienda, gasolinera & 19 & 13 & 68.4 \\
Otros servicios & 2 & 1 & 50.0 \\
Comercio prod. agrícolas & 18 & 10 & 55.5 \\
Otros & 1 & 1 & 100.0 \\
\hline Total & 237 & 135 & 57.0 \\
\hline
\end{tabular}

Fuente: Encuesta a socios, 1997.

De esta forma, por la falta de control en los préstamos, una proporción importante de los mismos se encuentran en cartera vencida $-57 \%$ - sin embargo, como se puede observar en el Cuadro 6, las diferencias en la recuperación, en una proporción importante de los proyectos, se debe fundamentalmente a las prácticas desarrolladas por los propios fondos para recuperar los créditos. En productos básicos, la cartera vencida es de 56\%, sin embargo, los recursos están orientados a la compra de semilla y fertilizantes, y la -muy reducida - totalidad de su producción, se dedica al consumo de la familia, por lo que se deduce que la mayoría de quienes lo recuperan, lo hacen con ingresos monetarios de otras fuentes distintas a las del préstamo.

Llama la atención que los proyectos de transporte, tienda, gasolinería y comercialización — que por la naturaleza de los negocios, deberían ser $100 \%$ recuperables_-, tengan niveles de cartera vencida superiores al promedio y mayores al de la ganadería y los frutícolas.

Por otra parte, cuando se realiza el pago, se encontró la siguiente dinámica. Al momento de preguntar a los beneficiarios acerca del origen de los recursos con los que cubren sus adeudos, se encontró que $60 \%$ lo hacía con los recursos generados por el proyecto, mientras que $40 \%$ restante lo hacía con otros de distinta procedencia. 
Cuadro 7

Número de apoyos recibidos por grupo

\begin{tabular}{lrc}
\hline Apoyos recibidos & Total & \% del Total \\
\hline No sabe & 9 & 3.79 \\
Una & 103 & 43.46 \\
Dos & 41 & 17.30 \\
Tres & 35 & 14.77 \\
Más de tres & 46 & 19.41 \\
No respuesta & 3 & 1.26 \\
\hline Total & 237 & 100.0 \\
\hline
\end{tabular}

Fuente: Encuesta a Socios, 1997

Lo anterior se debe a que los productores tienen como costumbre reciclar los recursos financieros en el propio predio, ya que llevan a cabo actividades múltiples; los ingresos monetarios que adquieren con una actividad los utilizan para financiar otra, así llega un momento en que los productores — por ejemplo — venden el ganado que engordaron con recursos del proyecto, pero en lugar de realizar el pago, los orientan a la cosecha de café, y con el ingreso por la venta de éste, cubren el adeudo si se les requiere, lo que da como resultado, que les sea difícil determinar el origen de sus recursos.

Otra práctica que resulta común es que, pese a las reglas de operación, se señala la necesidad de ampliar la cobertura. Se encontró que $43 \%$ de los grupos ha sido beneficiado una vez y $57 \%$ ha recibido más de un crédito. Esto se debe a que los montos de apoyo que brindan los fondos son muy reducidos. Se forma un grupo de interesados — que van pidiendo apoyo a nombre del mismo- , y entre ellos nombran a los responsables de cada proyecto, los cuales son los realmente beneficiados, por eso, para lograrlo, es necesario que la mayoría del grupo constituido pida varios financiamientos. Lo anterior ha propiciado que sólo en apariencia crezca la cobertura y que frecuentemente se viole la normatividad, sin que los participantes en el fondo se inconformen.

Para la asignación de recursos, los FRI exigen que los indígenas se organicen en grupos. Pese a lo que se esperaba, no se encontraron, en muchos casos, grupos organizados. Aunque los hay, la mayoría son de trabajo, comunidades y sociedades de solidaridad social, es decir, organizaciones que sólo requieren el consentimiento de sus socios, ponerle un nombre y registrarlo ante la Asamblea General. La mayoría de éstas son sujetos de crédito solamente de los organismos oficiales dependientes de SEDESOL.

\section{Organización de productores}

El hecho de que $76 \%$ de los entrevistados provenga de organizaciones sin registro legal reafirma la fragilidad de las mismas frente a la pregunta de cómo se conformó el grupo: $62.4 \%$ de los casos respondió que "en una asamblea", es decir, fue resultado de la necesidad 
de asociarse para acceder a recursos del fondo como éste lo exige, mientras que apenas $18 \%$ de los entrevistados refieren pertenecer a un grupo antes del apoyo.

Lo anterior no hace sino confirmar la naturaleza del propósito de asociación y también explica el reducido ritmo de consolidación de los Fondos.

Si los grupos se constituyen con el propósito exclusivo de acceder a un apoyo financiero, cuando éste se logra, se termina el elemento de cohesión y la organización tiende a desaparecer. Cuando se constituye con el fin de mejorar las condiciones de vida de los agremiados, tienden a consolidarse los factores de cohesión, definen estrategias para mejorar y las expresan en proyectos; asimismo, estimulan a los beneficiados a mantener la dinámica que permita la revolvencia de los Fondos, criterios honestos y transparentes para el manejo de los recursos y criterios sencillos para elegir los proyectos.

\section{Características de los proyectos}

Las principales peticiones de apoyo a proyectos productivos hasta 1997 fueron agrícolas, $55.7 \%$ y particularmente en la producción de básicos, $42 \%$. Le siguen en importancia los pecuarios, $18.1 \%$, de los cuales los bovinos representaron $12.2 \%$, y en tercer lugar se encuentran los de servicios.

En la mayoría de los casos se trata de proyectos de subsistencia, pero existen algunos con perspectiva comercial como son los de producción de café, hortalizas, flores y pesca, que pueden ser rentables, sin embargo, esta tendencia no se modificó sustancialmente hasta 1999.

No sólo encontramos diferencias en las asignaciones, sino en el tipo de proyectos apoyados. Los Fondos Regionales de Chiapas habían apoyado 2370 proyectos desde su fundación hasta 1997 en las siguientes actividades, entre los más importantes:

- $\quad$ Producción de básicos

- Ganadería bovina

- Tiendas

- Comercio de productos agrícolas

- $\quad$ Otro tipo de ganado

- Hortalizas

- Deshidratados

- Frutícolas

Sin embargo, separando el capital recibido por tipo de proyectos, se observan cambios que muestran un predominio de dos grupos.

Entre 1997 y 1999, se aprecia que, conforme los fondos tienen una mayor autonomía, tienden a orientar sus proyectos a la ganadería bovina, no obstante, siguen predominando los proyectos agrícolas y pecuarios. 
Cuadro 8

Evolución de los proyectos aprobados

\begin{tabular}{|c|c|c|}
\hline & 1997 & 1999 \\
\hline Agrícola & $43.1 \%$ & $40.4 \%$ \\
\hline Pecuario & $36.3 \%$ & $44.8 \%$ \\
\hline Agroindustrial & $5.0 \%$ & $4.0 \%$ \\
\hline Artesanal & $0.6 \%$ & $0.6 \%$ \\
\hline Otros & $15.0 \%$ & $10.2 \%$ \\
\hline Total & $100.0 \%$ & $100.0 \%$ \\
\hline Total en pesos & $\begin{array}{lll}59 & 108 & 212.00\end{array}$ & 59408880.00 \\
\hline
\end{tabular}

Fuente: INI.

En los primeros momentos en que los consejos directivos se veían muy influidos por los directores de los centros coordinadores indigenistas, los Fondos tendían a apoyar la producción de básicos y, en menor medida, de ganado bovino y agroindustriales como los frutícolas y hortícolas, sin embargo, la inexperiencia tanto en la producción, como en las formas organizativas de trabajo en común propiciaron que los proyectos fueran acaparados por unos cuantos socios, o que se fueran a la quiebra.

La gama de proyectos apoyados es tan diversa que al parecer no hubo líneas de financiamiento determinadas, sino que se otorgó a todo aquel que solicitó el productor o un grupo de éstos. Por otra parte, no se cuidó el principio de que fueran productivos y recuperables, ya que algunos se apoyaron de infraestructura, y una gran cantidad estaba orientada a mejorar la condición productiva de los indígenas, lo que se tradujo en un incremento de la producción - y probablemente en el consumo- pero no en la capitalización de los beneficiarios.

La autonomía de los socios provocó que — al ser Chiapas una entidad predominantemente montañosa - fuera el ganado bovino el que mayor peso tuviera en los apoyos: $12.2 \%$ en 1997, después de los agrícolas, cuando resulta frecuente que las zonas de explotación ganadera se ubiquen en zonas con pendientes de hasta 45 grados.

Otro aspecto que resulta relevante en el proceso de asignación de recursos es que una proporción muy importante de los apoyos se orientan a proyectos de subsistencia y no son productivos $\mathrm{y}$, por tanto, no necesariamente recuperables.

\section{Participación de los socios en los proyectos}

Contrario a la concepción que se tiene sobre las formas de trabajo en las comunidades indígenas (trabajo comunitario), la mayoría de los entrevistados aseguró que realiza las actividades del proyecto en forma individual (66.7\%), y que esta forma de trabajo fue acordada por el grupo y la costumbre (96.7\%), mientras que el trabajo por comisiones 
sólo representa $15.8 \%$. Al parecer, las actividades trabajadas en grupo se realizan en donde su naturaleza requiere mayor intensidad de trabajo, como en la producción de frutas, hortalizas, flores y apicultura.

En algunas actividades, para las que se requiere apoyo y en las que la agrupación de bienes resultaría la forma de organización óptima por la generación de economías de escala — como la ganadera - no se agrupan. La fuerza de la costumbre del sentido de propiedad provoca que se trabaje en forma individual — lo que resulta poco económico-, pero atiende perfectamente un problema cultural, su estatus. ${ }^{17}$

En los proyectos que absorben elevadas dotaciones de capital y requieren un pequeño número de operarios $(6.8 \%)$, como es el caso de las tortillerías, transportes, tiendas y gasolinerías, se asumen dos formas de participación en los proyectos. En una, el grupo nombra un comité administrador que frecuentemente termina quedándose con el bien, o se establecen secuencias mediante las cuales se turnan para atenderlo, lo anterior se debe al elevado número de beneficiarios y a las pocas ocupaciones que garantiza el proyecto. ${ }^{18}$

\section{Beneficios}

La mayoría de los socios entrevistados coincidió en indicar que el proyecto le trajo beneficios, ya que recibió recursos del fondo, sin embargo, por lo limitado de éstos, y por la falta de dirección de las líneas de negocios, 52\% no logró cumplir con sus objetivos, el mayor impacto del apoyo es que el socio logró trabajar en su comunidad sin necesidad de migrar.

Por otra parte, que $98.7 \%$ de los socios no reciba pago alguno por la actividad que realiza, y que $58 \%$ declare que no ha tenido ganancias, son indicadores de que los proyectos no han cumplido con sus propósitos.

No obstante, programas sociales como los FRI en Chiapas han tenido algunos efectos positivos. Entre 1996 y 1998, se redujo la población masculina que no recibe ingresos, ${ }^{19} \mathrm{de}$ $29 \%$ a $27 \%$, aunque aumentó la población femenina que no los recibe, de $31.6 \%$ a $37 \%$.

Sin embargo, descontando a los que no reciben ingresos, se redujo la población que gana menos de un salario mínimo, de $46 \%$ a $41 \%$, en el caso de los hombres, de $63 \%$ a $57 \%$ en el caso de las mujeres y aumentó la población ocupada que gana de 1 a 2 sML y de más de dos.

\footnotetext{
17 Si se tiene una vaca, la persona tiene un estatus en su comunidad, pero si esta vaca va a un hato común entonces la persona no adquiere el estatus que le significa ser su dueño.

18 Este fenómeno no es exclusivo de los FRI, se encontró también en proyectos apoyados por FOANES, es un fenómeno generalizado cuando se trata de apoyar grandes proyectos sociales donde intervienen muchas personas.

19 STYPS-INEGI, ENE, Chiapas, 1996 y 1998.
} 


\section{Conclusiones}

De acuerdo con lo anterior, se observa la necesidad de una revisión de la filosofía del programa, lo que implica modificaciones sustantivas en las políticas e instrumentos, así como organización de los participantes y los agentes gubernamentales. En particular, se requiere una definición precisa y directiva de la CNI como orientador y encauzador de los procesos de desarrollo económico y social de las comunidades indígenas.

- Se necesita, además de una revisión y ajuste de la normatividad, el uso de los instrumentos para la asignación de recursos. Por otra parte, se considera que las acciones de restructuración deben ser inducidas con programas en el corto y largo plazos, en los cuales, se orienten las acciones de los fondos por medio de metas de operación anuales con criterios específicos.

- Un aspecto por resolver y aceptar es que la comunidad no se asuma como organización y, por tanto, para ser considerada como sujeto de crédito. Por otra parte, se debe plantear una restructuración profunda de los FRI, establecer un marco legal donde queden claramente identificadas las funciones, obligaciones, atribuciones y responsabilidades de cada uno de los miembros del Consejo Directivo, pues en la normatividad de fondos no se indica ninguna de las anteriores.

- En el caso de la Comisión Financiera, deberá señalarse al responsable específico del manejo del dinero y al que habrá que pedirle cuentas. Asimismo, en el caso de que sea la comunidad la apoyada, será necesario la identificación de todos y cada uno de los beneficiarios directos y, por tanto, responsables de pagar la inversión.

- Al presentar una petición de apoyo de Fondos debería incluirse la lista de los beneficiarios directos con nombre completo, monto que le corresponde pagar a cada uno y su firma, avalado por las autoridades locales. Adicionalmente, las personas que reciban directamente el recurso tendrían responsabilidades jurídicas derivadas del crédito.

\section{Del sentido de propiedad}

Respecto al sentido de propiedad, este determina la autonomía, por ello, para fortalecer el de los socios y consolidar la posición de los Fondos como organismo financiero regional, es necesario desarrollar las siguientes estrategias, y acciones:

- Reforzar entre los organizaciones pertenecientes al Fondo, la percepción de que el apoyo, al ser propio y tener el fin de mejorar su condición productiva, debe ser reintegrado, ya que esto permitirá mantener el apoyo a los vecinos de su comunidad y para ellos mismos. Se les debe aclarar que el crédito es de propiedad comunitaria - y al ser reintegrados es posible que se continúen operando de esta forma - aun cuando se facilite de manera individual. 
- Desarrollar talleres de inducción a los solicitantes de recursos, en los que se les sensibilice acerca de la importancia de hacer un buen uso del crédito, desarrollando actividades con potencial productivo en la zona, con el propósito de que recuperen su inversión y obtengan un beneficio monetario o de producto.

- Establecer mecanismos que garanticen la recuperación al finalizar el producto o en su venta y solicitar garantías para generar corresponsabilidad en el pago del apoyo.

En el caso del Consejo Directivo es necesario fortalecer el sentido de propiedad entre sus miembros, y esto sólo se puede lograr, capacitándolos para que obtengan una mayor autonomía en las labores de gestión del Fondo, donde logren identificar sus principales problemas, formas sencillas de evaluación del manejo de los recursos, así como de elementos para la definición de líneas de inversión. La capacitación debe ser diferencial:

- A los miembros de la Comisión Financiera para que evalúen los reportes mensuales y cotejen notas de ejercicio, así como para verificar cierto tipo de gastos que muestren dudas sobre su manejo.

- A la Comisión Técnica para que identifiquen proyectos con potencial productivo en la zona de acuerdo con su vocación, y

- A los miembros de la Comisión de Evaluación para visitar los proyectos vencidos y valorar su condición.

Aunado a la capacitación, deberán establecerse criterios de responsabilidad para impedir el mal manejo de los recursos. Los Fondos Regionales Indígenas, deberían:

- Coordinar los esfuerzos institucionales con relación a las comunidades indígenas, en las que el INI tenga el poder de concertación y la representación y legitimidad para la toma de decisiones frente a las comunidades indígenas.

- Establecer políticas de tasa de interés, revolvencia y garantías de recuperación para que el fondo funcione como un instrumento de capitalización y desarrollo de comunidades indígenas.

- Implantar políticas de refinanciamiento de los Fondos al fijar compromisos o metas específicas de acuerdo con la filosofía económica y social del programa y con respecto al comportamiento financiero del Fondo, particularmente con referencia a la revolvencia de los fondos con el propósito de su saneamiento y desarrollo.

- Establecer políticas sobre la asignación de los recursos que precise y diferencie las condiciones del apoyo. Esas políticas deben distinguir entre el financiamiento a proyectos productivos, de infraestructura y sociales. En esta mezcla, el Fondo podría operar como detonador del desarrollo regional y local de las comunidades indígenas, lo que 
requiere un replanteamiento de la filosofía del programa en cuanto al fondo como instrumento de desarrollo económico y social.

Uno de los objetivos del programa es que fomente la creación y organización financiera de organizaciones y comunidades indígenas, con el propósito de que haya fondos o bolsas de recursos que garanticen su proceso de ahorro y capitalización. En la medida que dichos Fondos sean autosuficientes o tengan el potencial para acudir a otras fuentes de financiamiento, los recursos fiscales pueden ir disminuyendo en su apoyo. Por otra parte, las nuevas organizaciones requerirán de mayor ayuda y de un programa de mediano plazo.

Por otra parte, los programas de infraestructura y de desarrollo social no tienen que ser financiados por los Fondos, cuya principal función debe ser de instrumento de ahorro e inversión para capitalizar los proyectos productivos, que, a su vez, deben ser rentables y garantizar su revolvencia y crecimiento.

\section{De las recuperaciones}

Es necesario desarrollar estrategias que corresponsabilicen a los socios con la conservación del fondo como institución financiera de apoyo. Con el propósito de mejorar la recuperación de los recursos, es necesario corresponsabilizar a los miembros del Consejo Directivo en la operación financiera del fondo.

- Cada uno debe operar como detonador de los procesos de formación y desarrollo de organizaciones que se caractericen por la autogestión y autonomía con base comunitaria y local. Esto implica no sólo allegarse los recursos, sino capacitar e inducir los procesos de planeación, organización y participación a nivel comunitario, con el fin de que se logren los objetivos para los cuales fueron creados los fondos.

- Establecer estrategias de desarrollo microregional que impulsen la actividad productiva, el empleo y el ingreso de las comunidades indígenas y que consideren la inserción y la viabilidad del centro regional y el mercado que las orienta. Para ello, el fondo debe apoyarse en el Comité consultivo propuesto y en el levantamiento e identificación de necesidades y propuestas a nivel comunitario y por organización.

- Implantar formas de organización y participación en la planeación comunitaria que propicie la organización y contribución de la economía local, recogiendo necesidades e iniciativas, de tal manera que la planeación desde abajo a nivel local y comunitario se empate con la planeación desde arriba tanto cupular como la correspondiente al Consejo Consultivo mencionado. La forma que puede adoptar es similar a la de los Comités Coordinadores de Planeación Comunitaria (COCOPLA).

- Establecer criterios y políticas para los fondos con el objeto de que la asignación y el manejo de los recursos sean claros y permitan el logro de los objetivos del fondo. Es 
necesario indicar con claridad el carácter y la función de éste. La concepción de proporcionar capital y posteriormente crear los ahorros sin considerar la organización, planeación, capacitación y desarrollo tecnológico es imposible. Se necesita diferenciar los programas de apoyo social de los de desarrollo económico, señalando cuáles recursos son para transferencias y cuáles para capitalización, lo que requiere la revisión del programa mencionado.

Respecto a la organización, se requiere el cambio y revisión de la filosofía del programa con el fin de distinguir las diferencias entre el Fondo Regional y las organizaciones paralelas de éstos con figuras legales exclusivas para las organizaciones participantes, lo que limita cualquier acción de integración e inclusión y propicia clientelismo y corrupción. Es más, afecta la normatividad del Fondo y la subordina a los mandatos de la organización adoptada por las organizaciones que detentan el poder.

Lo que queda sin discusión es la búsqueda o modificación de instancias que financien pueblos y comunidades indígenas en la búsqueda de instancias productivas que ofrezcan empleo e ingresos regulares a esta población.

\section{Bibliografía}

INEGI-INI, Encuesta Nacional de Zonas Indígenas, 1997. INI-SEDESOL, Instituto Nacional Indigenista 19891994, México, 1994.

Sedesol, Reglas de operación de la Comisión Nacional para el Desarrollo de Pueblos y Comunidades Indígenas.
SEDESOL-INI-SOLIDARIDAD, Fondos Regionales de Solidaridad(Manual de Operación), México, INI, 1993, pp. 10-11.

www.cgap.org/docs/CaseStudy_centralamerica 